\title{
«Mehr koordinieren, mehr delegieren - mehr Freude»
}

Daniel Lüthi

Text und Bilder

danielluethi[at]gmx.ch
Vor dem Ärztehaus steht eine Schuttmulde. Der Sitz des FMH-Generalsekretariats in Bern wird renoviert, aus einer Wohnung wird Büroraum. Das Büro des Präsidenten ist das gleiche wie vorher, die Möbel haben zum Teil geändert. Die Uhr zeigt die Zeit jetzt berndeutsch an: «Zäa vor zwöi» meldet sie um 13 Uhr 50 zum Beispiel. Die tiefen Ledersessel aus der Ära de Haller hat Jürg Schlup durch Bürostühle ersetzen lassen, «die sind effizienter».

\section{Kulturwandel}

Die Renovation, die im Inneren der FMH inhaltlich im Gang ist, umschreibt Schlup immer wieder mit dem Begriff «Kulturwandel». Im Sitzungszimmer versinnbildlicht ihn der neue Präsident, indem er nicht oben am Tisch sitzt, sondern in der Mitte, «mal links, mal rechts, aber immer in der Mitte». Der Wandel sei ein Prozess, sagt Schlup. Konkret? «Ich will einen partnerschaftlichen, partizipativen Füh-

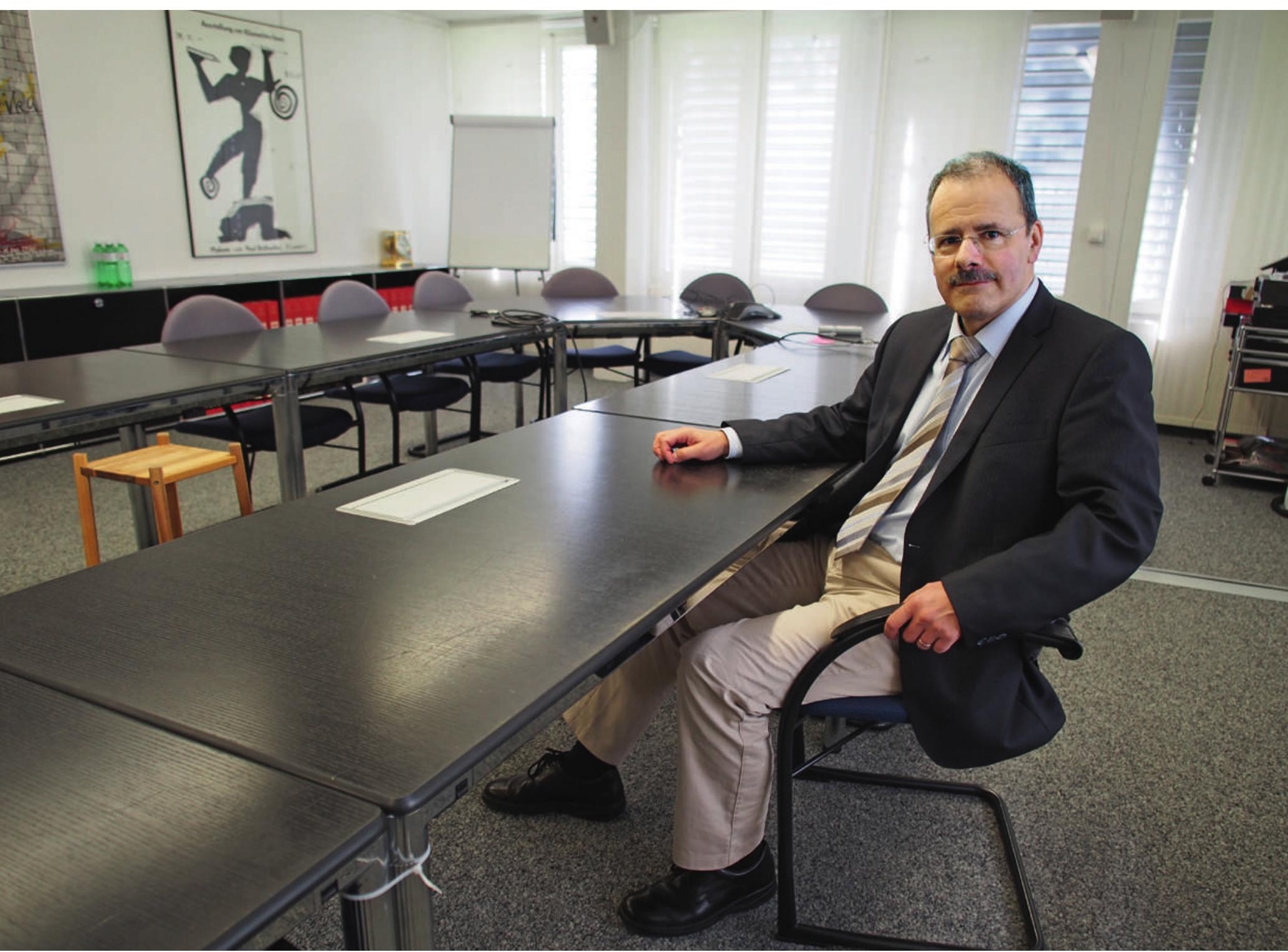


rungsstil pflegen. Ich will, dass man Konflikte bezeichnet und austrägt und so Lösungen näherkommt.» Als Beispiel nennt er das Gebiet der hochspezialisierten Medizin, «das ist eine Baustelle, da gab es in der Ärztezeitung kontradiktorische Artikel, die den Eindruck erweckten, hier sei ein Aufstand, eine Meuterei im Gang. Es geht um die Zentralisierung von Spitalleistungen, um das Thema ZZentrum versus Peripherie, eine komplizierte Sache.» Das sei das Schöne daran, sagt Schlup: «Es hat nur gemottet, nie geknallt. Wenige machten die Faust im Sack, sondern man sass zusammen und suchte nach Lösungen.» Konkret? Er verweist auf einen Artikel in der SÄZ, wo von einer gesamtschweizerischen Planung, einer interkantonalen Vereinbarung, von Entscheidungsprozessen und Vernehmlassungsfristen die Rede ist.

\section{Strukturwandel}

An der Wand hängt die farbige Druckgrafik eines Aargauer Künstlers. Es heisst «la décision», und das sei auch der Grund, warum er dieses Bild aufgehängt habe, sagt Jürg Schlup. Im Juni vor einem Jahr musste er selber schnell eine wichtige Entscheidung treffen, an der Ärztekammer, bei der es um die Nachfolge von Jacques de Haller ging, der nach acht Jahren nicht mehr in den FMH-Zentralvorstand gewählt wurde und damit als Präsident nicht mehr kandidieren konnte. «Ja, wir wollten Jacques de Haller abwählen», sagt Schlup. Warum genau, mag er nicht mehr erörtern, «ich will lieber nach vorne schauen als zurück.»

\section{«Das Gesundheitswesen ist im Umbau.»}

Plötzlich stand Schlup selber als möglicher Nachfolger zur Debatte, «meine Kandidatur war ein Ad-hoc-Entscheid», kommentiert er, «und meine Wahl auch für mich eine grosse Überraschung. Ich bin dort schon ein wenig auf die Welt gekommen.» Er wusste, dass er den ziemlich abrupten Wechsel von der hausärztlichen Praxis in einer Berner Vorortsgemeinde auf den Präsidentensessel der wichtigsten nationalen Ärztevereinigung zuerst seinem Praxis-Partner und auch seiner Ehepartnerin würde schmackhaft machen müssen. Der Antrieb, sich wählen zu lassen, sei diese Erkenntnis gewesen: «Es kann, es darf nicht sein, dass sich nichts ändert.» Denn: «Das Gesundheitswesen ist im Umbau. Uns steht ein Strukturwandel bevor, der in anderen Branchen bereits stattgefunden hat, Stichwort: Lädelisterben`. Für die Ärzteschaft heisst das: Wir müssen unsere Dienstleistung so anpassen, dass wir sie in einem veränderten Umfeld anbieten können.» Im ambulanten Sektor heisse das, erklärt Hausarzt und

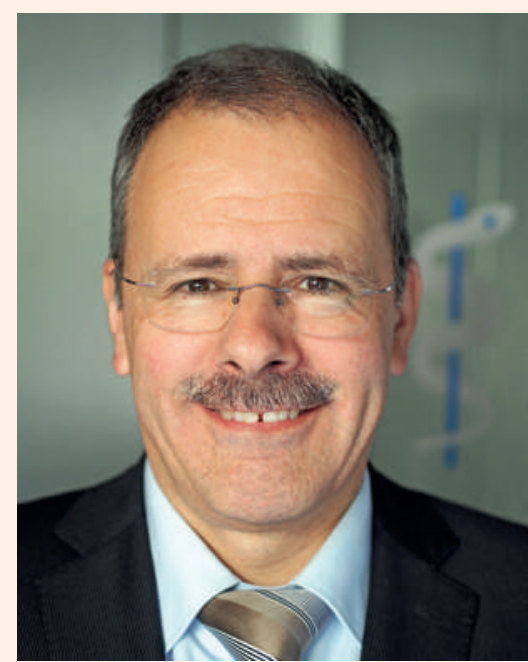

\section{Jürg Schlup}

Dr. med. Jürg Schlup wurde 1955 in Bern geboren. Er besuchte die Schulen in Köniz und absolvierte das Medizinstudium in Bern. Im Rahmen seiner Weiterbildung zum Facharzt Allgemeine Innere Medizin arbeitete er in den Fachbereichen Arbeitsmedizin, Chirurgie, Innere Medizin, Gynäkologie, Pädiatrie und Psychiatrie. An der Universität St.Gallen absolvierte er ein Nachdiplomstudium in Unternehmensführung, das er mit dem Executive MBA HSG abschloss. 24 Jahre lang arbeitete Schlup als Hausarzt in einer Doppelpraxis in Zollikofen bei Bern. Parallel dazu war er als Standespolitiker aktiv. 1984 bis 1987 war er Präsident des Verbandes der Assistenz- und Oberärzte des Kantons Bern, 2001 bis 2010 Präsident der Ärztegesellschaft des Kantons Bern, 2008 bis 2010 Vizepräsident der Spitalversorgungskommission des Kantons Bern. Im Juni 2012 wurde er überraschend zum neuen FMH-Präsidenten gewählt. Er trat sein Amt Anfang Dezember 2012 an. Jürg Schlup ist verheiratet und Vater von zwei erwachsenen Kindern. Er wohnt in Zollikofen bei Bern.

Gesundheitspolitiker Schlup: «Weg von der Einzelpraxis, hin zum interdisziplinären, ambulanten Gesundheitsversorgungszentrum.» Damit sei jedoch keineswegs eine Gruppenpraxis von mehreren Hausärzten gemeint, sondern eben ein interdisziplinäres Versorgungszentrum, zum Beispiel mit Haus- und Kinderärzten, Psychiatern und Physiotherapeutinnen, Gynäkologinnen und Medizinischen Praxisassistentinnen; bei einem grösseren Zentrum zusätzlich mit Apothekern, Pflegefachpersonen und auch ambulant tätigen Chirurgen. 
Ein ähnlicher Wandel sei auch im Spitalsektor im Gang, es gebe immer mehr kleinere Häuser, die sich spezialisieren. «Mehr koordinieren, mehr delegieren mehr Freude», ist Schlups Zusammenfassung dieser Entwicklung.

\section{Rahmenbedingungen}

Das FMH-Präsidium ist ein Vollamt, Schlup verabschiedete sich vor einem Jahr also von seiner Tätigkeit als Hausarzt und suchte für die Doppelpraxis in Zollikofen einen Nachfolger. «Zahlreiche Inserate brachten keinen Erfolg. Hohes Engagement, lange Arbeitszeiten und das Verhältnis zwischen Aufwand und Ertrag sind heute nicht besonders attraktiv. «Dank der Unterstützung eines Kollegen ist es mir gelungen, einen Internisten in einem Spital zu überzeugen, im Dezember in meine Praxis zu wechseln.»

So weit, so gut. Bloss: Geht es an einem, der Medizin studiert und diesen Beruf über zwanzig Jahre

\section{«Mein Beruf ist auch mein Hobby.»}

ausgeübt hat, wirklich spurlos vorbei, wenn er sein Sprechzimmer, seine Praxis verlassen muss? «Nein», sagt Schlup. «Der ganz direkte, persönliche Austausch und die Nähe in schwierigen Lebenssituationen fehlen mir. Ich vermisse meinen Laden manchmal schon. Jetzt bemühe ich mich um gute Rahmenbedingungen, damit unsere Mitglieder ihren Beruf unter guten Voraussetzungen ausüben können.» Hohes Engagement und lange Arbeitszeiten sind für ihn als Rahmenbedingungen geblieben: «Ich arbeite jetzt eher noch mehr als vorher, aber als Arzt bin ich mir gewohnt, Verantwortung zu tragen und Belastungen. Im Übrigen ist es ein Privileg, dieses Amt auszuüben. Es fordert, wie der Arztberuf auch, viel, gibt aber auch viel zurück.»

Privates hat daneben wenig Platz. «Mein Beruf ist auch mein Hobby», sagt Schlup. Ansonsten sei er, was seine Freizeit anbelange, bescheiden geworden. «Ab und zu mit Freunden ein Bier, manchmal eine Wanderung oder ein Ausflug mit der Frau. Ein Buch lesen - zu selten.»

Der Gefahr, sich selber zu wenig Beachtung zu schenken, sei er sich bewusst, stellt er nüchtern fest, «die Burnout-Gefährdung gehört zu unserer Berufs- gruppe. Als selbständig erwerbender KMUler hatte ich diesbezüglich eher mehr Gestaltungsmöglichkeiten.» Jetzt ist er 24 Stunden lang in ein System eingebunden, «ich muss rund um die Uhr erreichbar sein. Aber ich fühle mich nicht als Gefangener des Systems. Der ständige Austausch, den mir diese Aufgabe ermöglicht, ist auch eine Ressource. Zudem ist die Leistungsbereitschaft all meiner Mitarbeiterinnen und Mitarbeiter hier im Generalsekretariat und meiner Kolleginnen und Kollegen im Zentralvorstand sehr hoch und motivierend.»

\section{Zwischenbilanz}

Um die richtigen Diagnosen und Therapien gehe es nach wie vor, sagt Jürg Schlup, bloss auf einer anderen Ebene. Zwei seiner Stärken seien bei der Suche nach Lösungen besonders wichtig: «Ich bin kompromissbereit und konsensorientiert - bis zu einem gewissen Punkt.» Dass dies jedoch nicht immer die gewünschten Früchte trägt, musste Schlup bereits in seinem ersten Amtsjahr erfahren. Die Tarifverhandlungen mit dem Krankenkassenverband scheiterten. «Wir sind wegen ein paar umstrittenen Zeilen drei Wochen lang am Ort getreten, haben einmal gar von sechs Uhr abends bis halb zwei Uhr in der Früh gerungen - vergeblich. Das hat mich sehr enttäuscht. Jetzt darf via Bundesrat der Staat in den Tarif eingreifen, das ist ein schlechtes Zeichen für eine Partnerschaft.»

Lieber hält sich Jürg Schlup an positive Erlebnisse. «Die Geschichte mit dem Zulassungsstopp ist für uns jetzt, nach zehn Jahren - endlich - gut ausgegangen. Fünf Monate lang haben wir hinter den $\mathrm{Ku}-$ lissen hart gearbeitet - und mit viel Lobbying ein für die Ärzteschaft akzeptables Resultat erstritten: Wer mindestens drei Jahre an einer anerkannten schweizerischen Weiterbildungsstätte gearbeitet hat, für den gilt kein Zulassungsstopp.»

$\mathrm{Zu}$ streiten gehört für ihn jetzt, mehr denn je, dazu. Oder anders: die Auseinandersetzung mit Kritik. Die Holzskulptur, die ein austretendes Mitglied des Zentralvorstandes der FMH vor Jahren geschenkt hat, steht nach wie vor beim Eingang. Sie heisst «Das kritische Mitglied». «Ich habe für dieses Kunstwerk ein hübsches Schild machen lassen. Denn es erinnert mich jeden Tag daran, für wen wir da sind, wer meine Arbeit legitimiert - auch wenn der einzelne Mensch in der Regel nicht mehr, wie früher in der Praxis, direkt bei mir anklopft.»

\section{Die nächste «Begegnung mit ...»}

Am Ende jeden Monats stellt die Schweizerische Ärztezeitung eine Persönlichkeit vor, die sich im Gesundheitswesen engagiert. Im Dezember schildert Daniel Lüthi seine Begegnung mit Valérie D'Acremont, Tropenmedizinerin an der Medizinischen Universitätspoliklinik in Lausanne und am Swiss TPH in Basel. 